\title{
ASPECTO EPIDEMIOLÓGICO DAS NOTIFICAÇÕES DE MALÁRIA ENTRE 2012 A 2017 NO MUNICÍPIO RONDOLÂNDIA-MT
}

\author{
EPIDEMIOLOGICAL ASPECT OF NOTIFICATIONS OF MALARIA FROM 2012 TO 2017 IN \\ THE MUNICIPALITY RONDOLÂNDIA-MT
}

\author{
Gleison Faria ${ }^{a^{*}}$, Graciely dos Santos da Luz ${ }^{b^{*}}$, Thais Antunes Betin ${ }^{c^{*}}$ \\ gleisonfaria@hotmail.coma,grazy-luz@hotmail.comb', thaisbetin@hotmail.com \\ Faculdade de Ciências Biomédicas de Cacoal - FACIMED \\ Data de recebimento do artigo: 23/01/2019 \\ Data de aceite do artigo: 21/06/2019
}

\section{RESUMO}

A malária é uma doença infecciosa febril aguda, causada por protozoários transmitidos pela fêmea infectada do mosquito Anopheles. A cura é possível se a doença for tratada em tempo oportuno e de forma adequada. Contudo, a malária pode evoluir para forma grave e para óbito. Objetivo da pesquisa foi verificar a incidência da malária no município de Rondolândia - MT no período de 2012 a 2017. Materiais e Método - tipo transversal, documental, descritivo e quantitativa, as informações foram obtidas a partir da análise de dados epidemiológicos fornecidos pela vigilância em saúde do município de Rondolândia - MT, que foram atendidos na unidade básica de saúde Hueverton Bruno da Silva Rabelo que foram tabuladas através de um questionário realizados pelos próprios pesquisadores contendo 4 perguntas básicas de acordo com a ficha de notificação compulsória do SIVEP-Malária. Resultados - Foram notificados 177 pacientes de acordo com o período informado (20122017), sendo $14 \%$ dos casos positivos e $86 \%$ negativo, $100 \%$ dos casos acometeram o sexo masculino, $56 \%$ informaram que a atividade exercida (ocupação) é a pecuária. Conclusão - A doença ocasionada pelo Plasmodium vivax foi a de maior incidência no município de Rondolândia-MT. Os cinco anos investigados nessa pesquisa revelaram que o perfil epidemiológico da malária se manteve, acometendo sobretudo o sexo masculino $(\mathrm{n}=25)$. Obteve uma média anual de 4.16 casos/ ano uma incidência da doença dentro do território de RondolândiaMT de 0,69 casos em cada 100 habitantes comparado ao último censo.

Palavras-chave: Malária, evolução, conduta

\section{ABSTRACT}

Malaria is an acute febrile infectious disease caused by protozoa transmitted by the infected female of the Anopheles mosquito. Cure is possible if the disease is treated in a timely and appropriate manner. However, malaria can progress to severe form and death. The objective of the study was to verify the incidence of malaria in the municipality of Rondolândia - MT from the period of 2012 to 2017. Materials and Method - transverse type, documentary, descriptive and quantitative, the information was obtained from the analysis of epidemiological data provided by surveillance in in the municipality of Rondolândia - MT, which were attended at the Hueverton basic health unit, Bruno da Silva Rabelo, which were tabulated through a questionnaire carried out by the researchers themselves containing 4 basic questions according to the compulsory notification form of SIVEPMalaria. Results - A total of 177 patients were reported according to the reporting period (2012-2017), 14\% of the cases were positive and $86 \%$ were negative, $100 \%$ of the cases were male, $56 \%$ reported that the activity was occupation livestock. Conclusion - The disease caused by Plasmodium vivax was the one with the highest incidence in the municipality of Rondolândia-MT. The five years investigated in this research revealed that the epidemiological profile of malaria remained, mainly affecting males $(n=25)$. It obtained an annual average of 4.16 cases / year an incidence of the disease within the territory of Rondolândia-MT of 0.69 cases in every 100 inhabitants compared to the last census.

Keyword: Malaria, Evolution, Conduct. 


\section{Introdução}

A malária é considerada uma doença de notificação compulsória, pois todos os casos que são suspeitos ou mesmo confirmados em órgãos públicos ou privados são obrigatoriamente notificados as autoridades de saúde, as quais são usadas as fichas de notificações e investigação ${ }^{1}$.

A malária, mundialmente um dos mais sérios problemas de saúde pública, é uma doença infecciosa causada por protozoários do gênero Plasmodium e transmitida ao homem por fêmeas de mosquitos do gênero Anopheles, caracterizase como uma doença infecciosa, apresentando um quadro febril agudo, causado pelo. Quatro espécies de plasmódio podem causar a doença: $\mathrm{P}$. falciparum, P. vivax, P. malariae e P. ovale ${ }^{1}$.

A cura da doença é possível, se o paciente procurar tratamento em tempo oportuno e de forma adequada, caso não tratada pode evoluir para formas mais graves chegando a levar a pessoa a obito ${ }^{2}$.

Dados da Organização Mundial da Saúde (OMS) mostram que seu impacto sobre as populações humanas continua aumentando: ocorre em mais de 90 países, pondo em risco cerca de $40 \%$ da população mundial - estima-se que ocorram de 300 a 500 milhões de novos casos, com média de um milhão de mortes por ano. Representa, ainda, risco elevado para viajantes e migrantes, com casos importados em áreas nãoendêmicas ${ }^{1}$.

No Brasil, o maior número de casos é registrado na região Amazônica, cujas condições ambientais e socioculturais favorecem a expansão de sua transmissão. Em 2003, 407.691 casos da doença foram notificados na Amazônia Legal (divisão política do território nacional que engloba nove estados: Amazônia, Acre, Amapá, Maranhão, Mato Grosso, Pará, Rondônia, Roraima e Tocantins). Pela intensidade da transmissão destacaramse os estados do Amazonas, Rondônia e Pará, responsáveis por $50 \%$ da totalidade dos casos de malária no país, com uma incidência parasitária anual, respectivamente, de 46,3/1.000 habitantes, 64,4/1.000 habitantes e 17,6/1.000 habitantes. Em toda a Amazônia, as infecções causadas pelo P. vivax (79\%) prevaleceram sobre as do P. falciparum $(21 \%)^{1}$.
No ano de 2014 houveram 143.552 novos casos de malária notificados no Brasil, destes, 4.593 corresponderam a casos importados de países que fazem fronteira com Brasil e outros 32 países. Neste mesmo ano apenas o estado de Rondônia apresentou aumento no número de casos de malária falciparum. Por outro lado, os estados do Acre, Amazonas, Amapá, Mato Grosso e Roraima apresentaram sinalização de surto, bem como 112 (14\%) dos municípios da região ${ }^{3,4}$.

A malária é considerada uma das doenças mais antigas do mundo, representando um desafio para a saúde pública. A doença está relacionada a pobreza, suas incidências esta correlacionada a grupos vulneráveis, sendo de extrema importância a vigilância em saúde e fazer o monitoramento populacional ${ }^{5}$.

O estado de Mato Grosso no ano de 2005 registrou 9.774 casos da doença correspondendo a um percentual de $2 \%$ do total dos casos da Amazônia legal. Em relação ao ano de 2004 o estado de Mato Grosso apresentou um aumento de $38,7 \%{ }^{6}$ o percentual muito grande, Dessa Forma o objetivo dessa pesquisa foi informar a incidência dos casos de malárias ocorridas entre 2012 a 2017 no município de Rondolândia - MT, divisa com o estado de Rondônia.

\section{Materiais e Métodos}

Antes de iniciar a pesquisa foi encaminhado para o comitê de ética e pesquisa (CEP), da Faculdade de ciências biológicas de Cacoal-RO (FACIMED por meio da sua aprovação recebeu o número do parecer consubstanciado $\mathrm{n}^{\circ}$ 2.813.060.

O presente trabalho trata-se de um estudo documental retrospectivo, transversal, com análise descritiva e abordagem quantitativa ${ }^{7,8}$. A pesquisa foi realizada no município de Rondolândia-MT, teve como instrumento de coleta de dados um questionário semiestruturado de 05 perguntas de múltiplas escolhas que foram extraídos da própria ficha de notificação da doença abordando critérios de inclusão com as seguintes variáveis: Identificar o perfil do paciente quanto a faixa etária, sexo, cor, escolaridade, zona de ocorrência e atividade exercida, com intuito de direcionamento da coleta de dados, as quais foram extraídas as informações 
secundárias do banco de dados do Sistema de Vigilância Epidemiológica- SIVEP que foram fornecidas pela secretaria municipal de saúde do município, setor de vigilância em saúde pelo servidor responsável, disponibilizados para os pesquisadores em forma de planilhas no Microsoft Excel $^{\oplus}$.

Para os dados obtidos não houveram custos, remuneração e nem exposição da integridade dos pacientes notificados, não foi necessário o uso do termo de consentimento livre esclarecido - TCLE pois os dados foram secundários sendo assim solicitado a dispensa do mesmo ao $\mathrm{CEP}^{7,8}$.

A pesquisa teve início no mês de agosto e setembro de 2018, e obteve uma amostra de 25 notificações da doença. Não houve nenhuma exclusão de notificação, pois antes de receber os dados foram encaminhados via e-mail para a vigilância em saúde exatamente os dados necessários para a pesquisa, sendo assim, facilitando para o responsável do setor de vigilância em saúde a filtração do banco de dados do SINAN, os quais após foram disponibilizados de acordo como solicitado para os pesquisadores ${ }^{7,8}$.

A estatísticas utilizadas na tabulação dos dados obtidos foram: frequência absoluta e frequência relativa. Tiveram como critério de exclusão todas as notificações que não tinham informações necessárias para o estudo e não pertencentes ao município de Rondolândia-MT ou os casos não encerrados no período investigado.

\section{Resultados}

A Tabela 1 apresenta a amostra de 25 pacientes que tiveram resultados positivos que confirmaram o diagnóstico da malária, sendo todas as notificações do sexo masculino, faixa etária entre 18 a 59 anos de idade. As notificações dos casos positivos foram entre os anos de 2012 a 2017, sendo o de maior relevância o ano de 2015 com $(60 \%)$ dos casos 8 .

Tabela 1 - Perfil sociodemográfico dos pacientes, Rondolândia-MT - 2012 - 2017.

\begin{tabular}{|c|c|c|c|c|c|c|c|c|c|c|c|c|c|c|}
\hline DADOS & \multicolumn{2}{|c|}{2012} & \multicolumn{2}{|c|}{2013} & \multicolumn{2}{|c|}{2014} & \multicolumn{2}{|c|}{2015} & \multicolumn{2}{|c|}{2016} & \multicolumn{2}{|c|}{2017} & \multicolumn{2}{|c|}{ TOTAL } \\
\hline Sexo & $\mathrm{N}$ & $(\%)$ & $\mathrm{N}$ & $(\%)$ & $\mathrm{N}$ & $(\%)$ & $\mathrm{N}$ & $(\%)$ & $\mathrm{N}$ & $(\%)$ & $\mathrm{N}$ & $(\%)$ & $\mathbf{N}$ & $\%$ \\
\hline Masculino & 4 & 16 & 6 & 24 & - & - & 15 & 60 & - & - & - & - & 25 & 100 \\
\hline Feminino & - & - & - & - & - & - & - & - & - & - & - & - & & \\
\hline \multicolumn{15}{|l|}{$\begin{array}{c}\text { Faixa etária } \\
\text { (idade) }\end{array}$} \\
\hline$<18$ & - & - & - & - & - & - & 2 & 8 & - & - & - & - & & \\
\hline $18-59$ & 2 & 8 & 5 & 32 & - & - & 13 & 52 & - & - & - & - & 25 & 100 \\
\hline$>60$ & 2 & 8 & 1 & 4 & - & - & - & - & - & - & - & - & & \\
\hline \multicolumn{15}{|l|}{$\begin{array}{c}\text { Escolaridade } \\
\text { (anos de estudo } \\
\text { concluídos) }\end{array}$} \\
\hline Nenhum & - & - & 1 & 4 & - & - & - & - & - & - & - & - & & \\
\hline 1 a 3 & 2 & 8 & 1 & 4 & - & - & 2 & 8 & - & - & - & - & & \\
\hline 4 a 7 & 1 & 4 & - & - & - & - & - & - & - & - & - & - & & \\
\hline 8 a 11 & - & - & - & - & - & - & 7 & 28 & - & - & - & - & 25 & 100 \\
\hline 12 a mais & - & - & - & - & - & - & - & - & - & - & - & - & & \\
\hline Não se aplica & - & - & - & - & - & - & - & - & - & - & - & - & & \\
\hline Ignorado & 1 & 4 & 4 & 16 & - & - & 6 & 24 & - & - & - & - & & \\
\hline
\end{tabular}


A Tabela 2, apresenta a tabulação dos dados, consta-se que $60 \%$ das notificações ocorreram no ano de 2015.

Tabela 2 - informações referentes ao ano de ocorrência Rondolândia-MT - 2012 - 2017.

\begin{tabular}{ccccc}
\hline ANO & \multicolumn{2}{c}{ LOCALIDADE } & \multicolumn{3}{c}{ Total } \\
\hline & URBANO & RURAL & $\%$ & $\mathrm{~N}$ \\
2012 & 2 & 2 & 16 & 4 \\
2013 & 1 & 5 & 40 & 10 \\
2014 & - & - & - & \\
2015 & - & 11 & 44 & 11 \\
2016 & - & - & - & \\
2017 & - & - & - & \\
& & Total Geral & $\mathbf{1 0 0}$ & $\mathbf{2 5}$
\end{tabular}

Fonte: SIVEP/SEMUSA/MS, 2012-2017, Rondolândia-MT

A Tabela 3 apresenta, conforme a tabulação, 56\% das notificações da doença ocorreu em pecuarista.

Tabela 3 - Atividades exercidas pelas vítimas - Rondolândia-MT - 2012 - 2017.

\begin{tabular}{|c|c|c|c|c|c|c|c|c|c|c|c|c|c|c|}
\hline & & & & & & & & & & & & & \multicolumn{2}{|c|}{ Total } \\
\hline DADOS & \multicolumn{2}{|c|}{2012} & \multicolumn{2}{|c|}{2013} & \multicolumn{2}{|c|}{2014} & \multicolumn{2}{|c|}{2015} & \multicolumn{2}{|c|}{2016} & \multicolumn{2}{|c|}{2017} & $\mathbf{N}$ & $\%$ \\
\hline Atividade & $\mathrm{N}$ & $\%$ & $\mathrm{~N}$ & $\%$ & $\mathrm{~N}$ & $\%$ & $\mathrm{~N}$ & $\%$ & $\mathrm{~N}$ & $\%$ & $\mathrm{~N}$ & $\%$ & & \\
\hline Agricultor & 3 & 12 & - & - & - & - & - & - & - & - & - & - & 3 & 12 \\
\hline Pecuarista & - & - & - & - & - & - & 14 & 56 & - & - & - & - & 14 & 56 \\
\hline Domestica & - & - & - & - & - & - & - & - & - & - & - & - & & \\
\hline Turismo & - & - & - & - & - & - & - & - & - & - & - & - & & \\
\hline Garimpeiro & - & - & - & - & - & - & - & - & - & - & - & - & & \\
\hline Exploração vegetal & - & - & 4 & 16 & - & - & - & - & - & - & - & - & 4 & 16 \\
\hline Caça/pesca & - & - & - & - & - & - & - & - & - & - & - & - & & \\
\hline $\begin{array}{c}\text { Construção estrada/ } \\
\text { barragem }\end{array}$ & - & - & - & - & - & - & - & - & - & - & - & - & & \\
\hline Mineração & - & - & - & - & - & - & - & - & - & - & - & - & & \\
\hline Viajante & - & - & - & - & - & - & - & - & - & - & - & - & & \\
\hline Outros & 1 & 4 & 1 & 4 & - & - & - & - & - & - & - & - & 2 & 8 \\
\hline Ignorado & - & - & 1 & 4 & - & - & 1 & 4 & - & - & - & - & 2 & 8 \\
\hline TOTAL DE CASOS & & & & & & & & & & & & & 25 & 100 \\
\hline
\end{tabular}

Fonte: SIVEP/SEMUSA/MS, 2012-2017, Rondolândia-MT 


\section{Discussão}

Com relação a faixa etária das vítimas, ambos os sexos e idade estão propícios a contrair a doença, entre outros fatores, a atividade laboral contribua para uma maior exposição do sexo masculino, devido seu trabalho direto no campo".

No ano de 2013 e 2014 a maior índice de tratamento para a doença foi detectada na etnia indígena. Em 2014 o menor foi entre os indivíduos negros ${ }^{3}$.

Essa incidência com o passar dos anos pode haver um aumento, se não tiver avanço em tratamento ou de prevalência contra o vetor, poderá ter mais números de vítimas. $\mathrm{O}$ investimento em treinamento para as equipes do município e de (extrema) importância, pois a área territorial e é bem pequena, sendo possível a realização de um bom trabalho na zona rural e urbana.

O município de Rondolândia-MT, apresentou durante os cinco anos de pesquisa, 14\% dos casos positivos, sendo todos eles do sexo masculino com idade entre 18-59 anos, e $88 \%$ deles residentes na zona rural do município ${ }^{8}$.

A exposição do corpo para contrair a doença está muito relacionada ao trabalho, o qual se desenvolve na maior parte no período de atividade do mosquito, principalmente na população rural, ou seja, próximo a campos fechados, sendo assim podendo potencializar o risco de transmissão a malária ${ }^{10,11}$.

O município de Rondolândia-MT apresentou de 2012 a 2017177 notificações de suspeitas de malária, sendo, $14 \%(\mathrm{n}=25)$ casos (positivos) e $86 \%(n=152)$ (negativos). Por se tratar de uma doença compulsória, é obrigatória a notificação no banco de dado (SIVEP-MALÁRIA) ${ }^{12,13}$ sendo ela positiva ou negativa conforme afirma o decreto 78.231 , de 12 de agosto de $1976{ }^{1}$.

As notificações ocorreram entre os anos de 2012 e 2017, obtendo (maior) incidência em (menor) incidência nos anos de 2014, 2016 e 2017, comportamento relacionado ao déficit de recursos humanos capacitados no município para realização dos exames e também por ser uma região cercada de propriedades particulares (fazenda), a maioria das vítimas acabam se deslocando para os municípios mais próximos como: (Andreaza-RO, Ji-Paraná-RO e Cacoal$\mathrm{RO})^{8}$.
De acordo com a OMS (2017) "Estima-se que em 2016 houve cinco milhões de casos a mais de malária do que em 2015. Já os óbitos pela doença ficaram em torno de 445 mil, número similar ao do ano anterior" 14 .

De acordo com a pesquisa, $56 \%$ das notificações com resultado positivas tem como ocupação no dia-a-dia a pecuária (criadores de gado de corte e leiteiro), $60 \%$ dos casos foram notificados pertencentes ao município de Rondolândia -MT, $24 \%$ do município de Colniza-MT $4 \%$ ao município de Conservam-MT e $12 \%$ ao estado de Rondônia, todos esses pacientes que foram notificados através da ficha do SIVEP-Malária são moradores do município de Rondolândia ${ }^{15}$. O município de Rondolândia é cercados por fazendas e também várias aldeias indígenas que fazem divisa com vários territórios de municípios do estado de Mato Grosso e Rondônia sendo eles: (Colniza, Aripuanã e Juína no estado de Mato Grosso e Andreaza, Ji-Paraná e Cacoal no estado de Rondônia) ${ }^{16}$.

A aproximação do vetor/homem/ambiente, mostrou-se complexa em diferentes ocupações, ou seja, requer mais conhecimento de planejamento e ações intersetoriais capazes de contribuir para o controle da doença no município ${ }^{19}$.

Com base no estudo, não se pode afirmar o local exato onde tem maior incidência de casos notificados, pelo próprio comprometimento da pesquisa, mas de acordo com a ficha de notificação do SIVEP-Malária do município de RondolândiaMT, tem vários casos pertencentes a uma fazenda denominada "Fazenda Castanhal" que fica nas redondezas do município ${ }^{8,12,13}$.

A sintomatologia da doença é clássica, podendo apresentar hipertermia (> temperatura), calafrios, tremores, sudorese e cefaleia apresentados de forma conjunta em muito dos casos. Alguns pacientes antes de apresentar sintomas classifico da malária, apresentam sintomas simples como náuseas, vômitos, cansaço e a falta de apetite ${ }^{2}$. Existem aqueles pacientes que vivem em áreas endêmicas que podem ter contraído a doença por diversas vezes e o seu organismo desenvolver mecanismo de resistência ou imunidade parcial, sendo assim, esse indivíduo pode apresentar sintomas da doença ${ }^{20}$. 
O profissional deve obter o máximo de informações possíveis com detalhamento sobre as viagem e rotina do paciente, pois é possível o paciente morar em um determinado local, mas ter passado algum final de semana em uma área endêmica e ter contraído a doença ${ }^{10,11}$.

Esses casos acontecem muito no município de Rondolândia-MT, devido a extensão territorial $\left(12.670,852 \mathrm{~km}^{2}\right)$, cercado de fazendas que chegam a ficar $180 \mathrm{~km}$ de distância da zona urbana sendo elas:-fazenda panela, castanhal, morro grande, santa Silva e rio madeirinha ${ }^{16}$.

A sintomatologia grave apresentada pela doença-é caracterizada pelo surgimento de alguns sintomas em conjunto como: alteração de consciência, prostração, dispneia ou hiperventilação, hipotensão arterial, choque hemorrágicos, convulsões entre outros sinais ${ }^{2}$.

Antes de submeter o paciente ao tratamento, é realizado o exame de detecção da doença pelo profissional capacitado chamado de "gota espessa". Esse exame e bem simples, e usado uma gota de sangue colhida diretamente por punção digital ou venosa sem anticoagulante ${ }^{21}$.

No município de Rondolândia-MT, existe somente um profissional treinado e atuante na realização dos exames da malária. O município é pequeno e possui 3.854 habitantes ${ }^{16}$, ou seja, possui apenas uma unidade básica de saúde que atende a população com atendimentos 24 horas. Todos os exames após serem realizados pelo profissional, são e arquivados e passam por fiscalização detalhada, que ocorre cerca de $1 \mathrm{x} /$ ano, pela equipe (responsável) que desloca-se do escritório regional de saúde do município de Pontes e Lacerda-MT, os quais são supervisionados pelo laboratório central de saúde pública de mato grosso - LACEM/Cuiabá-MT².

O exame da malária chamado de gota espessa é o método oficial adotado pelo ministério da saúde para realização do diagnóstico da malária. Apesar do avanço de outras técnicas de diagnosticar a malária, o mesmo ainda continua sendo o método mais simples, de baixo custo, eficaz e de fácil realização e também foi reconhecido o método padrão pela Organização mundial de saúde $\mathrm{e}^{10,11,14}$.

Após confirmação do exame, o resultado é encaminhado para o médico da unidade, que imediatamente orientará o paciente a utilização dos medicamentos gratuitos conforme protocolo do ministério da saúde ${ }^{2}$.

Para a realização do tratamento da doença e é essencial correlacionar alguns fatores importantes como: identificar a espécie do protozoário infectante, a idade do paciente, condições associadas como: ser o paciente é do sexo feminino e se a mesma esta grávida e se faz algum tratamento de saúde ${ }^{2,20}$.

Existe diversos tipos de malária no Brasil, sendo elas: Plasmodium falciparum, Plasmodium vivax, Plasmodium malariae e Plasmodium ovale ${ }^{21}$. O Plasmodium falciparum é a espécie de malária mais agressiva, porque ela se multiplica rapidamente na corrente sanguínea. Já o Plasmodium vivax é um tipo de doença mais branda, que geralmente atinge pouco as hemácias, porém fica alojado por mais tempo no fígado ${ }^{22}$.

No município de Rondolândia-MT, o tipo de malária responsável pelos casos é o Plasmodium vivax $(<+/ 2,+/ 2,+,++,+++,++++$ cruzes $)$ e Plasmodium falciparum $(<+/ 2,+/ 2,+,++$, ,+++++++ cruzes). A malária ocasionada pelo Plasmodium falciparum é considerada-uma emergência médica e seu tratamento deve ser iniciado nas primeiras 24 horas. A malária do tipo Plasmodium vivax apresenta sintomas que se assemelham com a dengue, principalmente ao considerar a plaquetopenia, por esse motivo, é comum o retardamento do diagnóstico ${ }^{8}$.

Para a malária do tipo de maior gravidade ocasionado pelo Plasmodium falciparum são recomendadas as associações dos antimaláricos (artesunato+mefloquina/3 dias e primaquina/dose única), (quinina/ 3 dias + doxiciclina/ durante 5 dias e primaquina no $\left.6^{\circ} \mathrm{dia}\right)^{10,11}$. Uma vez que as medicações usadas para as malárias benignas não têm efeitos terapêuticos sobre a malária de maior gravidade, devido à resistência adquirida ${ }^{10,11,25}$.

$\mathrm{O}$ protocolo de tratamento seguido pelo município de Rondolândia-MT, é o disponível no rodapé da ficha de notificação do SIVEP-Malária (item 1 a 3) ${ }^{12,13}$ e são prescritos de acordo com o tipo de malária: Plasmodium vivax com "Cloroquina" em 3 dias e "Primaquina" em 7 dias; Infecções por Plasmodium falciparum com "Quinina" em 3 dias + "Doxiciclina" em 5 dias + "primaquina" no $6^{\circ}$ dia; Infecções mistas por Plasmodium vivax +Plasmodium falciparum com "mefloquina" em dose única e "primaquina" em 7 dias ${ }^{8}$. 
A incidência da malária com resultados tpositivos'no município de Rondolândia -MT foi de 6,48 casos/mil habitantes. Comparando com os dados de 2012 a 2017 com média de 5 casos/ ano, não foram notificados nenhum óbito pela doença ${ }^{25}$.

O estudo da incidência da malária no município de Rondolândia-MT, demonstrou que as informações de saúde do município, contribuise para o entendimento da epidemiologia da doença no estado de Mato Grosso, sobretudo contextualizando os seus valores determinados. Busca-se para o futuro próximo planejar e programar atividades de prevenção e controle da doença junto com a equipe da unidade básica de saúde do município (gestores, agentes de endemias, agentes comunitários de saúde, vigilância em saúde) sendo assim, desenvolver um trabalho que busque determinar os riscos, monitorar e avaliar a doença e criar intervenções direcionadas para o controle do vetor

Como medidas de prevenção individual que poderiam ser orientados através de palestras educativas nas escolas do município como: o uso de mosquiteiros impregnados ou não com inseticidas, roupas confortáveis que protejam braços e as pernas e o uso de repelente, nas casas inserir nas portas e janelas telas de proteção para evitar que os mosquitos entre para dentro da residência no período noturno.

Como medidas de prevenção coletivas com obrigatoriedade pelos gestores do município, a construção de drenagem de águas, saneamento básico para eliminação do criadouro do mosquito, limpeza das margens, aterros de lugares alagados com suspeita de proliferação do vetor, supervisionamento de vegetação aquáticas que podem servi de habitat de reprodução.

\section{Considerações Finais}

Os cinco anos investigados nessa pesquisa revelaram que o perfil epidemiológico da malária se manteve, acometendo sobretudo o sexo masculino $(n=25)$. Obteve uma média anual de 4.16 casos/ ano uma incidência da doença dentro do território de Rondolândia-MT de 0,69 casos em cada 100 habitantes comparado ao último censo do IBGE 2010. Os dados também mostraram que a doença ocasionada pelo Plasmodium vivax foi a de maior incidência no município de Rondolândia-MT, desencadeando uma série de sintomas clássicos como hipertermia, tremores e cefaleia.

Amaláriaeumgrandeproblemadesaúdepública que precisar ser notificado, diagnosticado e tratado o mais rápido possível com acompanhamento adequado, os diagnósticos rápidos diminuem a letalidade relacionada à enfermidade, a divulgação desta publicação visa aumentar a efetividade da vigilância epidemiológica e prevenir a ocorrência da doença nas áreas não-endêmicas ou de baixa endemicidade como forma de controle da doença em todo o mundo.

\section{Agradecimentos}

Os autores agradecem à Secretaria Municipal de Saúde de Rondolândia-MT, pelo fornecimento dos dados, e ao responsável pela realização dos exames de malária do município.

\section{Referências}

1. Brasil, Ministério da Saúde- Manual de diagnóstico laboratorial da malária- Ministério da saúde: BrasíliaDF; 2005 [acesso em 15 mar 2018] Disponível em: bvsms. saude.gov.br/bvs/publicacoes/malaria_diag_manual_ final.pdf

2. Brasil, Ministério da saúde - Malária - Brasília-DF; 2017a [acesso em 12 mar 2018] Disponível em: https://portalms. saude.gov.br/saude-de-a-z/malaria

3. Brasil, Ministério da Saúde - Boletim epidemiológico Brasília -DF; 2017d [acesso em 15 abr 2018] Disponível em: http://portalarquivos2.saude.gov.br/images/ pdf/2015/agosto/18/2015-009---Mal--ria-para-publica---o.pdf

4. Brasil, Ministério da Saúde - Boletim epidemiológico Brasília -DF; 2017d [acesso em 15 abr 2018] Disponível em: http://portalarquivos2.saude.gov.br/images/ pdf/2015/agosto/18/2015-009---Mal--ria-para-publica---o.pdf

5. Brasil, Ministério da Saúde. Vigilância em saúde. Ações inovadoras e resultados - Brasília-DF; 2014 [acesso em 14 mar 2018] Disponível em: http://portalarquivos2.saude. gov.br/images/pdf/2015/janeiro/22/relatorio-gestao-svs2011-2014-preliminar.pdf 
6. Brasil, Ministério da saúde. Sistema nacional de vigilância em saúde. Relatório de situação Mato Grosso, Brasília-DF; 2005 [acesso em 14 mar 2018] Disponível: http://bvsms. saude.gov.br/bvs/publicacoes/relatorio_snvs_mt_2ed.pdf

7. Gil, ACNF. - Como elaborar projetos de pesquisa. 3 ed. São Paulo - SP; 2001.

8. Semusa - Secretaria municipal de saúde de RondolândiaMT. Setor vigilância em saúde. Dados epidemiológicos da Malária no município - Rondolândia-MT; 2018.

9. Couto, R.A., Latorre, MRDO., Santi, SM., Natal, D. Malária autóctone notificada no estado de São Paulo: aspectos clínicos e epidemiológicos de 1980 a 2007 . Rev Soc Bras Med Trop. 2010;43(1):52-8.

10. Brasil, Ministério da Saúde - Secretaria de Vigilância em Saúde -Departamento de Vigilância EpidemiológicaGuia prático de tratamento da malária no Brasil Brasília- DF, 2010.: Ministério da Saúde; 36p. (Série A. Normas e Manuais Técnicos).

11. Brasil, Ministério da Saúde, Secretaria de Vigilância em Saúde, Departamento de Vigilância Epidemiológica. Guia de Vigilância Epidemiológica: Caderno 10 - Malária. 7. ed. Brasília, 2010: Ministério da Saúde;

12. Brasil,MinistériodaSaúde-Superintendênciadevigilância em saúde - Ficha de notificação/investigação da malária - Brasília-DF; 2018 [acesso em 21 ago 2018] Disponível em: http://www.saude.mt.gov.br/suvsa/arquivo/795/ ficha-de-notificacao-investigacaodocumento

13. Brasil, Ministério da saúde - Orientações para o preenchimento do SIVEP-Malária - Brasília-DF, 2018[online] - Disponível: <http://bvsms.saude.gov.br/bvs/ folder/orientacoes_preenchimento_sivep_malaria.pdf $>$ Acesso 21/08/2018

14. Organização mundial da saúde - Organização Panamericana da saúde - Relatório da OMS mostra que houve aumento de casos de malária no mundo - Brasília, DF, Brasil, 2017 - [online] - Disponível: <https:// www.paho.org/bra/index.php?option $=$ com_conten t\&view $=$ article $\& i d=5557$ :relatorio - da - oms - mostra que-avancos-na-resposta-mundial-a-malaria-estaoparalisando\&Itemid=812>- Acesso 21/04/2018

15. Parente, AT - Incidência de malária no estado do Pará e suas relações com a variabilidade climática regional [dissertação]. Belém-PA, 2008: Universidade Federal do Pará. Instituto de Geociências;. 99p.

16. Ibge - Instituto Brasileiro de Geografia e Estatística Divisão Territorial do Brasil e Limites Territoriais - 2018 -[internet] - Disponível: <http://ftp://geoftp.ibge.gov.br/ Organizacao/Divisao_Territorial/2008/DTB_2008.zip> 1 de julho de 2008. Consultado em 11 de outubro de 2008 - Acesso em 21/06/2018.
17. Monteiro, MRCC; Fernandes, SC.; Ribeiro, MC. Aspectos clínicos e epidemiológicos da malária em um hospital universitário de Belém, Estado do Pará, Brasil, 2013 - [online] - Disponível: <scielo.iec.gov.br/pdf/rpas/ v4n2/v4n2a05.pdf - Rev Pan-Amaz Saude 2013; 4(2):3343 - Acesso 21/07/2018

18. Oliveira, EC. Fatores associados à distribuição da malária em área de assentamento, município de Juruena/MT [dissertação]. Cuiabá: Universidade Federal de Mato Grosso, Instituto de Saúde Coletiva; 2009.

19. Maciel, GBML; Espinosa, MM; Atanaka-Santos, M. Epidemiologia da malária no município de Colniza, Estado de Mato Grosso, Brasil: estudo descritivo do período de 2003 a 2009. Epidemiol. Serv. Saúde, Brasília, 2013;22(3):465-474. Disponível em: http://scielo. iec.gov.br/scielo.php?script=sci_arttext\&pid=S167949742013000300011\&lng=es\&nrm=iso

20. Mioto, LD., Galhardi, LCF., Amarante, MK. Aspectos parasitológicos e imunológicos da malária, Departamento de Ciências Patológicas, Universidade Estadual de Londrina, Biosaúde, Londrina, 2012;14(1) [acesso em 25 ago 2018] Disponível em: http://www.uel.br/ccb/ patologia/portal/pages/arquivos/.../BS_v14_1_DEF_42. pdf

21. Brasil, Ministério da Saúde. Manual de Diagnostico Laboratorial da Malária. Brasília-DF; 2009 [acesso em 17 mar 2018] Disponível em: http://https://www. infectologia.org.br/admin/zcloud/principal/2016/06/ manual_diagnostico_malaria.pdf

22. Agência Fiocruz e notícias, Ministério da Saúde. Malária - Manguinhos, Rio de Janeiro; 2013. [acesso em 21 jul 2018] Disponível em: https://agencia.fiocruz.br/malária

23. Laiola, CCP., Silva, CJM., Tauil, P.L. - Controle da malária no Brasil: 1965 a 2001 - Organización Panamericana de la Salud -Brasília-DF; 2002. [acesso em 15 jun 2018] Disponível em: https://www.scielosp.org/article/ rpsp/2002.v11n4/235-244/

24. Braga, E.M., Fontes, C.J.F., - Plasmodium - Malária. In: Neves, D. P. Parasitologia Humana. 10 ed. São Paulo: Atheneu; 2000. pp. 128-146.

25. Alves, A., Martins A., Adolphsson, S., Bockorny, B., Carleti, G., Cabral G., Souza, ACP., Vianna, A. - Malária grave importada. Rev. Bras. Ter. Intensiva, São Paulo, 2007;19(2):231-236.

\section{Como citar este artigo:}

Faria G, Luz GS, Betin TA. Aspecto epidemiológico das notificações de Malária de 2012 a 2017 no município de Rondolândia-MT. Rev. Aten. Saúde. 2019;17(60):83-90 